\title{
Capture and Detection of Plasmodium vivax Lactate Dehydrogenase in a Bead-Based Multiplex Immunoassay
}

\author{
Eric Rogier, ${ }^{1 \star}$ Doug Nace, ${ }^{1}$ Dragan Ljolje, ${ }^{1,2}$ Naomi W. Lucchi, ${ }^{1}$ Venkatachalam Udhayakumar, ${ }^{1}$ and Michael Aidoo ${ }^{1}$ \\ ${ }^{1}$ Malaria Branch, Division of Parasitic Diseases and Malaria, Centers for Disease Control and Prevention, Atlanta, Georgia; ${ }^{2} \mathrm{CDC}$ Fellowship, \\ Centers for Disease Control and Prevention, Atlanta, Georgia
}

\begin{abstract}
Laboratory detection of malaria antigens has proved valuable for research and epidemiological purposes. We recently developed a bead-based multiplex antigen assay for pan-Plasmodium and Plasmodium falciparum targets. Here, we report integration of a Plasmodium vivax-specific target to this multiplex panel: $P$. vivax lactate dehydrogenase (PvLDH). Within the multiplex panel, assay signal for purified PvLDH antigen titrated into the single-digit picogram range. Against a panel of polymerase chain reaction (PCR)-confirmed samples from acute $P$. vivax infections $(n=36)$, sensitivity was $91.7 \%$ in using PvLDH detection for identifying the presence of parasites. Specificity against a panel of persons with no Plasmodium infection $(n=44)$ was $100 \%$, and specificity against a panel of PCR-confirmed $P$. falciparum, Plasmodium malariae, or Plasmodium ovale infections $(n=164)$ was $90.2 \%$. Addition of this PvLDH capture and detection system into the multiplex antigen panel will now allow for sensitive screening for species identification of both $P$. falciparum and $P$. vivax in the laboratory.
\end{abstract}

Detection of malaria antigens produced during blood-stage infection has proven to be useful for both malaria diagnostics (using point-of-care rapid diagnostic test, RDT) and malaria research purposes (through laboratory assays). ${ }^{1-3}$ These field and laboratory assays benefit from well-defined antibodies raised against these antigens with high specificity in binding to the defined target and detection sensitivity within the nanogram to picogram range. ${ }^{4-6}$ In the laboratory, screening for the presence of malaria antigens (as a proxy for acute Plasmodium infection) outlines a more practical methodology over more labor-intensive and expensive nucleic acid-based assays. ${ }^{7}$ Whole blood, plasma, serum, or samples eluted from filter paper are all viable specimens for malaria antigen detection, and the flexible nature of this immunoassay allows large numbers of field samples to be processed in the laboratory in a high-throughput manner. ${ }^{7}$ Our group has previously designed a laboratory multiplex test for malaria antigens on the bead-based Luminex ${ }^{\circledR}$ platform and included targets for pan-Plasmodium aldolase and lactate dehydrogenase (LDH), as well as Plasmodium falciparum-specific histidine-rich protein 2 (HRP2). ${ }^{3,4}$ By deciphering the antigen profile for an individual's biological sample, it could be ascertained if any Plasmodium infection was present at the time of sampling and if the infection was $P$. falciparum. By having three targets on this multiplex panel, with each generating a "positive" or "negative" signal, eight total combinations (a +/- signal for three targets: $2^{3}$ ) were possible for a person's antigen profile, providing a snapshot of information regarding Plasmodium species presence and estimated parasite density. 4,7

We report here the expansion of this multiplex panel to include a Plasmodium vivax-specific target enzyme which is unable to be deleted from the genome: $P$. vivax lactate dehydrogenase (PvLDH). For laboratory experiments, the panPlasmodium LDH (pLDH) and PvLDH monoclonal antibodies were procured from Fitzgerald (Cambridge, United Kingdom; all raised in mouse), and coupling conditions were optimized with the Luminex Antibody Coupling kit (Luminex Corp,

\footnotetext{
* Address correspondence to Eric Rogier, Malaria Branch, Division of Parasitic Diseases and Malaria, Centers for Disease Control and Prevention, 1600 Clifton Rd., Atlanta, GA 30333. E-mail: erogier@ cdc.gov
}

Austin, TX) as described previously. ${ }^{4,7}$ For anti-pLDH (clone M1209063) and anti-PvLDH (clone M1709Pv2) conjugation to different bead regions (MagPlex beads, Luminex Corp, Austin, $\mathrm{TX}$ ), coupling was performed at an antibody concentration of $12.5 \mu \mathrm{g} / 12.5 \times 10^{6}$ beads. The detection antibodies consisted of a $1.0 \mathrm{mg} / \mathrm{mL} 1: 1$ antibody mixture, made of clones M1709Pv1 and M86550 that were previously biotinylated by the ThermoScientific EZ-link Micro Sulfo-NHS-Biotinylation Kit according to the manufacturer's protocol. Along with LDH detection, conjugated beads and detection antibodies were also included on each assay plate for HRP2 and pan-Plasmodium aldolase, and the immunoassay was run under the same conditions with same reagents as described previously. ${ }^{4}$ Assay plates were run on a MAGPIX machine (Luminex Corp) by collection of the median fluorescence intensity (MFI) signal for a target of 50 beads/region. Subtracting the MFI for background wells (exposed only to sample diluent) from sample MFI signal provided an MFI minus background (MFI-bg) signal used for analysis. Recombinant PfLDH and PvLDH antigens were kindly provided by MicroCoat (Starnberger See, Germany). Samples $(n=200)$ with or without PCR-confirmed malaria infection from travelers returning to the United States have been described previously, ${ }^{4}$ and their use has received ethical approval from the CDC Human Subjects Office (2017-192).

In making a dilution series of the purified recombinant antigens, only the capture antibody against pLDH was able to detect the PfLDH antigen, whereas the capture antibody specific for PvLDH provided only a nominal signal for the highest PfLDH concentration of $20,480,000 \mathrm{pg} / \mathrm{mL}$ (average MFI-bg of 32 on scale from 0 to 27,000) (Figure 1). However, for the PvLDH antigen, both capture antibodies were able to provide an assay signal titrating into the single-digit picogram range. Because the multiplex assay will be run on human samples in future studies, we ran a panel of human blood samples without Plasmodium infection $(n=44)$ to determine a reliable assay signal that would determine a positivity threshold for antigen presence and allow for a limit of quantitation (LOQ) (Figure 2A). From these 44 noninfected samples, the lognormal mean plus five SDs MFI-bg signal for the pLDH bead was 85, and for the PVLDH bead was 33. By extrapolating from the standard curves, this equals an LOQ for the pLDH bead of $190.0 \mathrm{pg} / \mathrm{mL}$ for the 

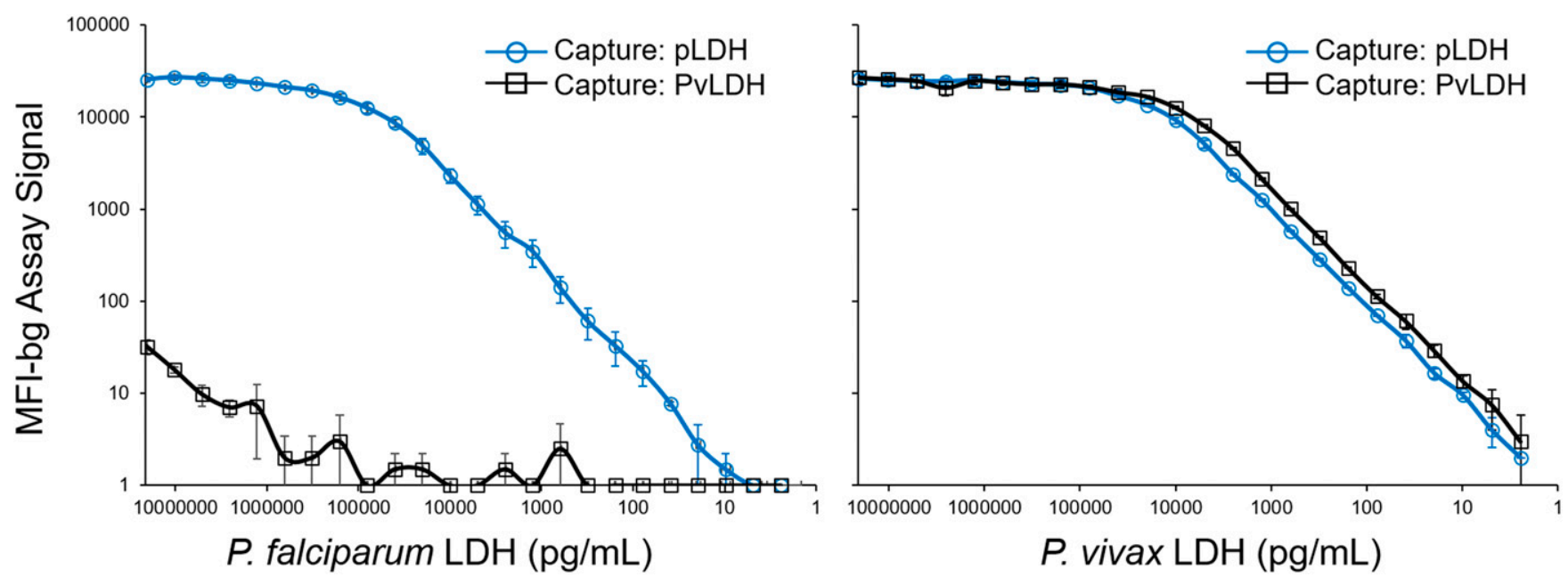

FiguRE 1. Detection of Plasmodium falciparum and Plasmodium vivax lactate dehydrogenase (LDH) by multiplex bead assay. Panels displayed for serial dilution series of both isoforms of LDH from 20,480,000 to $2.4 \mathrm{pg} / \mathrm{mL}$. Assay signal specific for pan-Plasmodium LDH (pLDH) capture/ detection is shown in blue lines with circles, and assay signal specific for $P$. vivax $\mathrm{LDH}(\mathrm{PvLDH})$ capture/detection shown in black lines with squares. Titration curves show average of results from two independent runs with error bars indicating SD.

PfLDH antigen and $56.8 \mathrm{pg} / \mathrm{mL}$ for the PvLDH antigen. For the PvLDH bead, this equals an LOQ for PvLDH of $7.2 \mathrm{pg} / \mathrm{mL}$.

To further examine sensitivity and specificity of the PVLDH bead against known Plasmodium acute infections, a panel of 200 plasma samples with known infection status by PCR were selected $^{4}$ and included $148 P$. falciparum, four Plasmodium malariae, 12 Plasmodium ovale, and $36 P$. vivax infections (Figure 2B). The pLDH bead was able to provide high MFI-bg signals for both $P$. falciparum and $P$. vivax infections, but typically a much lower signal for $P$. malariae and $P$. ovale. The PvLDH bead for the same samples provided high signals for more than $90 \%$ of $P$. vivax-infected plasma, whereas the MFIbg signals were nonexistent for the other three human malarias with the exception of $16 P$. falciparum infections found to have a positive PvLDH signal. From the bead-specific LOQ thresholds established previously, the specificity of the PvLDH bead against noninfected samples was $100 \%$ and was 90.2\% against other non-vivax Plasmodium infections with 17 of the 164 showing a positive MFI-bg signal by the LOQ threshold. Sensitivity of the PVLDH bead for detecting PCRidentified $P$. vivax infections was $91.7 \%$ with 33 of the 36 samples being bead assay positive with MFI-bg values typically $>20,000$ (29/36 samples, $80.6 \%)$.

With the addition of this $P$. vivax-specific target to the multiplex panel, the combinations of antibody positivity profile are now increased to $16\left(2^{4}\right)$. Many of these interpretations would likely be redundant with similar (or the exact same) explanations for why a person would have specific malaria antigen profile at the time of sampling, but this will need to be investigated further with field studies in different human and parasite populations. Of particular importance will be areas of $P$. falciparum and $P$. vivax coendemicity in assessing both the population prevalence of single infections with either species and coinfections with both. ${ }^{8-10}$ Interestingly, 16 of the 148 (10.8\%) PCR-identified $P$. falciparum infections also gave a positive signal for PvLDH. Although most of these were very low signals hovering around the MFI-bg threshold (10 of these had a MFIbg $<200$ ), four of these gave MFI-bg values $>1,000$. The potential may also exist for isoforms of PfLDH produced by different global strains, which would cross-react with the anti-PvLDH antibodies. Another possibility exists for true Pf/ Pv-mixed infections that were simply missed by the PCR assay. Further investigation of mixed malaria infections may require more sensitive nucleic acid assays to truly assess the performance of PVLDH detection in this multispecies context. Importantly, none of the malaria-negative blood samples provided a positive signal for PvLDH (or pLDH), so large population-level screening of blood samples that will largely be malaria negative would not be predicted to give many false-positive hits. Detection of the recombinant PvLDH antigen with the pLDH bead was more sensitive when directly compared with the detection of the PfLDH antigen with the same bead. This may suggest that the current anti-LDH monoclonal antibodies are overall more successful in discovering low-density $P$. vivax infections versus low-density $P$. falciparum, but this will ultimately need to be evaluated on extensive panels of human specimens to verify. Fortunately, detection of the HRP2 antigen has been shown by our group and others to be a very sensitive marker of low-density $P$. falciparum infections. ${ }^{2,4,5,7,11}$

Given that the pLDH bead typically gave low signals for the $P$. malariae and $P$. ovale infections, we will need to rely more on the pan-Plasmodium aldolase signal for the detection of these two malarias, and the multiplex assay has previously been shown to accomplish this as aldolase was found to be detected at lower concentrations when compared with pLDH. ${ }^{4}$ Although species-specific markers for $P$. malariae, $P$. ovale, and the zoonotic malarias are not currently included on this multiplex antigen panel, being able to confidently establish if $P$. falciparum and/or $P$. vivax is present may assist in narrowing down further nucleic acid assays to confirm presence and species of other Plasmodia. With more sensitive malaria laboratory tests being developed recently, the prevalence of $P$. malariae and $P$. ovale has been found to be at unexpectedly high levels in some settings, ${ }^{12,13}$ and this should be explored further to assess the impact on population health and malaria elimination strategies.

Unfortunately, to date, very few monoclonal antibodies have been raised against different malaria antigen targets. 

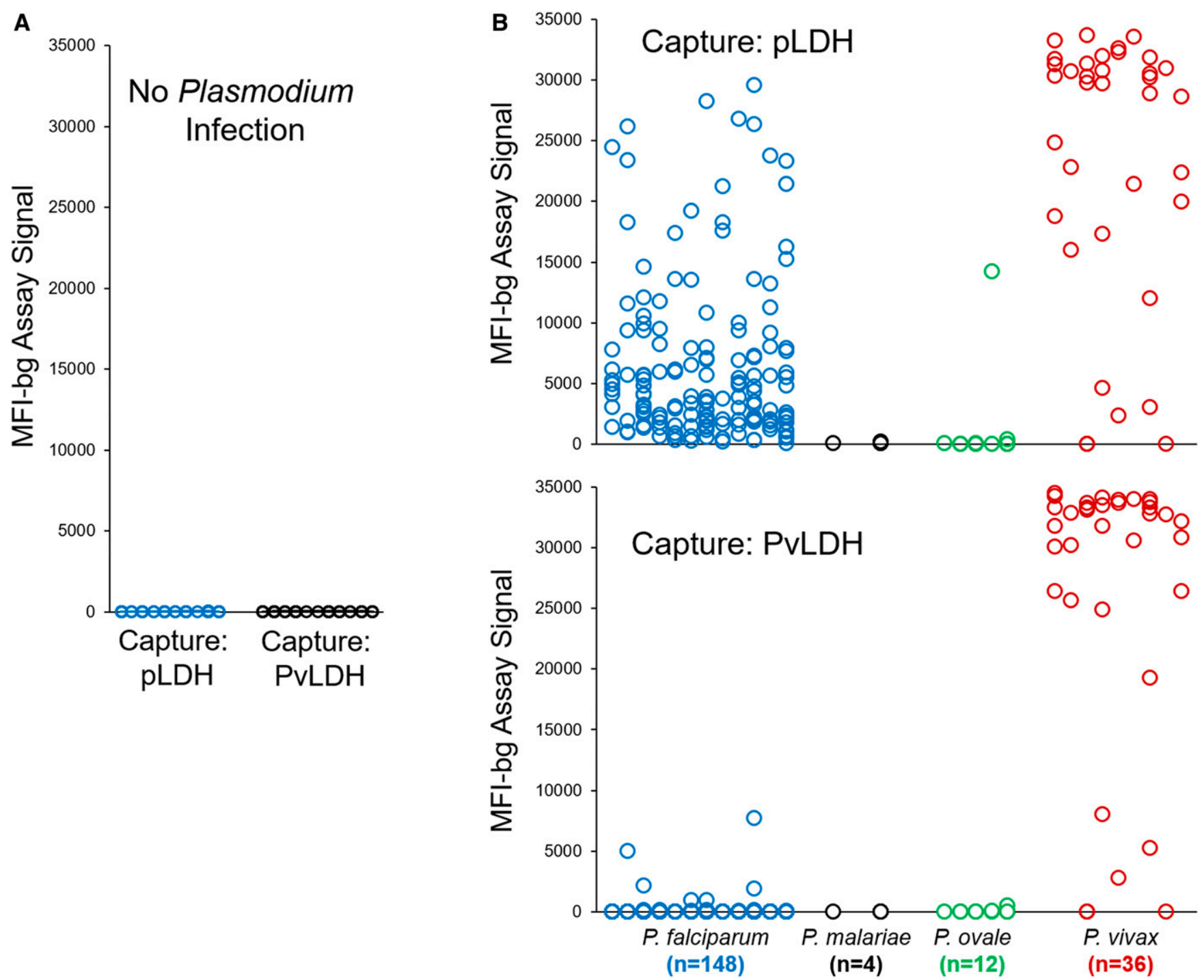

FIGURE 2. Assay signal for Plasmodium lactate dehydrogenase (pLDH) and $P$. vivax LDH (PvLDH) detection when testing samples from persons with no detectable Plasmodium infection or infection with one of the four human malarias. Multiplex bead assay signal shown for U.S.-based blood donors with no detectable Plasmodium infection $(n=44)(\mathbf{A})$ or an infection with one of the human malarias (B) Plasmodium falciparum $(n=148$, blue circles), P. malariae ( $n=4$, black circles), P. ovale ( $n=12$, green circles), or Plasmodium vivax ( $n=36$, red circles) as confirmed by PCR.

With the discovery of deletions in the Pfhrp2 and Pfhrp3 in natural $P$. falciparum populations that would render HRP2based diagnostics inadequate, ${ }^{14}$ renewed interest (especially in the point-of-care diagnostic field) has been garnered in generating new monoclonals, which would recognize targets unable to be deleted from the parasite genome. ${ }^{15,16}$ With the addition of each new capture/detection antibody to the multiplex laboratory assay, the resolution of interpretation for an individual's antigen profile will be further refined to allow a better prediction of Plasmodium status for that person at the time of sampling.

Received October 17, 2019. Accepted for publication January 28, 2020.

Published online March 2, 2020.

Acknowledgment: We acknowledge Mateusz Plucinski for manuscript review and editing.

Disclaimer: The findings and conclusions in this report are those of the authors and do not necessarily represent the official position of the CDC.
Authors' addresses: Eric Rogier, Doug Nace, Dragan Ljolje, Naomi W. Lucchi, Venkatachalam Udhayakumar, and Michael Aidoo, Malaria Branch, Division of Parasitic Diseases and Malaria, Centers for Disease Control and Prevention, Atlanta, GA, E-mails: erogier@cdc.gov, dnn4@cdc.gov, wou3@cdc.gov, frd9@cdc.gov, vxu0@cdc.gov, and mha3@cdc.gov.

\section{REFERENCES}

1. Boyce MR, O'Meara WP, 2017. Use of malaria RDTs in various health contexts across sub-Saharan Africa: a systematic review. BMC Public Health 17: 470.

2. Plucinski MM, Candrinho B, Dimene M, Colborn J, Lu A, Nace D, Zulliger R, Rogier E, 2019. Assessing performance of HRP2 antigen detection for malaria diagnosis in Mozambique. $J$ Clin Microbiol 57: e00875-9.

3. Plucinski MM, McElroy PD, Dimbu PR, Fortes F, Nace D, Halsey ES, Rogier E, 2019. Clearance dynamics of lactate dehydrogenase and aldolase following antimalarial treatment for Plasmodium falciparum infection. Parasit Vectors 12: 293. 
4. Plucinski MM et al., 2019. Screening for Pfhrp2/3-deleted Plasmodium falciparum, non-falciparum, and low-density malaria infections by a multiplex antigen assay. J Infect Dis 219:437-447.

5. Jang IK et al., 2019. Simultaneous quantification of Plasmodium antigens and host factor C-reactive protein in asymptomatic individuals with confirmed malaria by use of a novel multiplex immunoassay. J Clin Microbiol 57: e00948-18.

6. Plucinski $\mathrm{M}$ et al., 2017. Malaria surveys using rapid diagnostic tests and validation of results using post hoc quantification of Plasmodium falciparum histidine-rich protein 2. Malar J 16: 451.

7. Rogier $\mathrm{E}$ et al., 2017. Bead-based immunoassay allows subpicogram detection of histidine-rich protein 2 from Plasmodium falciparum and estimates reliability of malaria rapid diagnostic tests. PLoS One 12: e0172139.

8. Mitra S, Abhilash K, Arora S, Miraclin A, 2015. A prospective study from south India to compare the severity of malaria caused by Plasmodium vivax, P. falciparum and dual infection. J Vector Borne Dis 52: 281-286.

9. Jiram Al, Ooi CH, Rubio JM, Hisam S, Karnan G, Sukor NM, Artic MM, Ismail NP, Alias NW, 2019. Evidence of asymptomatic submicroscopic malaria in low transmission areas in Belaga district, Kapit division, Sarawak, Malaysia. Malar J 18: 156.

10. Tadesse F, Fogarty AW, Deressa W, 2017. Prevalence and associated risk factors of malaria among adults in East Shewa zone of Oromia regional state, Ethiopia: a cross-sectional study. BMC Public Health 18: 25.

11. Jang IK et al., 2018. A new highly sensitive enzyme-linked immunosorbent assay for the detection of Plasmodium falciparum histidine-rich protein 2 in whole blood. Malar J 17: 403.

12. Camargo-Ayala PA, Cubides JR, Nino $\mathrm{CH}$, Camargo $\mathrm{M}$, Rodriguez-Celis CA, Quinones T, Sanchez-Suarez L, Patarroyo ME, Patarroyo MA, 2016. High Plasmodium malariae prevalence in an endemic area of the Colombian Amazon region. PLoS One 11: e0159968.

13. Woldearegai TG, Lalremruata $A$, Nguyen $T T$, Gmeiner $M$, Veletzky L, Tazemda-Kuitsouc GB, Matsiegui PB, Mordmuller B, Held J, 2019. Characterization of Plasmodium infections among inhabitants of rural areas in Gabon. Sci Rep 9: 9784.

14. Gendrot M, Fawaz R, Dormoi J, Madamet M, Pradines B, 2019. Genetic diversity and deletion of Plasmodium falciparum histidine-rich protein 2 and 3 : a threat to diagnosis of $P$. falciparum malaria. Clin Microbiol Infect 25: 580-585.

15. Mouatcho JC, Goldring JP, 2013. Malaria rapid diagnostic tests: challenges and prospects. J Med Microbiol 62: 1491-1505.

16. Tao $D$ et al., 2019. A saliva-based rapid test to quantify the infectious subclinical malaria parasite reservoir. Sci Transl Med 11: eaan4479. 